\title{
Desarrollo del pensamiento crítico y creativo con estrategias motivacionales virtuales en una institución primaria en Perú
}

\author{
Mg. Marcela Tapia Caruajulca \\ ttapiaca8@ucvvirtual.edu.pe \\ https://orcid.org/0000-0001-7397-2239 \\ Dr. Aurelio Ruiz Pérez \\ aruizpe@ucvvirtual.edu.pe \\ https://orcid.org/0000-0001-7684-3475 \\ Programa Académico de Doctorado en Educación \\ Escuela de postgrado \\ Universidad César Vallejo \\ Chiclayo - Perú
}

\section{RESUMEN}

El trabajo de investigación tuvo como objetivo crear un modelo de estrategias motivacionales virtuales para el fortalecimiento del desarrollo del pensamiento crítico y creativo (PCC) de las niñas del V ciclo, de educación primaria de una institución pública, se orientó por el tipo básica, diseño no experimental, de carácter descriptiva propositiva, con enfoque cuantitativo. Se consideró el uso de herramientas o entornos virtuales de aprendizaje como motivación. Los datos fueron obtenidos por medio de una encuesta administrada por un cuestionario con 30 ítems, aplicada con la finalidad de identificar el nivel de desarrollo del PCC en el cual se ubicaron las estudiantes. Los medios electrónicos formulario Google y WhatsApp permitieron tomar la muestra a las niñas, valorados con escala Likert. Los resultados señalaron que la mayoría de las estudiantes que participaron se encuentran en el nivel de logro inicio y proceso; con esta información se elaboró la propuesta del modelo de estrategias, validado por juicio de expertos, con la pretensión de dar solución a la problemática encontrada además de permitir lograr estudiantes con características de pensadoras hábiles, sofisticadas con mejoras en sus decisiones, actuaciones y calificaciones.

Palabras clave: estrategias motivacionales; desarrollo de pensamiento; pensamiento crítico; pensamiento creativo. 


\title{
Development of critical and creative thinking with virtual motivational strategies in a primary institution in Peru
}

\begin{abstract}
The research work aimed to create a model of virtual motivational strategies for strengthening the development of critical and creative thinking of girls in the V cycle, of primary education of a public institution, was guided by the basic type, non-experimental design, descriptive-propositional, with a quantitative approach. The use of virtual learning tools or environments was considered as motivation. The data was obtained through a survey administered by a questionnaire with 30 items, applied to identify the level of development of critical and creative thinking in which the students were located. The electronic means Google and WhatsApp form allowed the girls to take the sample, valued with a Likert scale. The results indicated that most of the students who participated are at the beginning and process level of achievement; with this information, the proposal of the strategy model was elaborated, validated by expert judgment, with the aim of solving the problem encountered as well as allowing students to achieve characteristics of skillful, sophisticated thinkers with improvements in their decisions, actions, and qualifications.
\end{abstract}

Keywords: motivational strategies; thought development; critical thinking; creative; thinking

Artículo recibido: 15 enero 2022 Aceptado para publicación: 08 febrero 2022 Correspondencia: ttapiaca8@ucvvirtual.edu.pe Conflictos de Interés: Ninguna que declarar 


\section{INTRODUCCIÓN}

Los cambios generados a raíz de la calamitosa Covid-19 está suscitando caos en la educación a tal punto de revolucionar la educación, es más que indispensable formar "niños desde temprana edad con capacidades superiores, que sirvan como base para su vida", (Tapia \& Castañeda, 2021) por lo que es preciso tener que cambiar la rutina docente de realizar clases en un aula presencial a un aula virtual sincrónica o asincrónica, donde se tuvo que reinventar haciendo uso de la tecnología para lograr un aprendizaje integral que conduzca al estudiante a desarrollar su pensamiento crítico y creativo, habilidades fundamentales para tomar mejores decisiones y enfrentar los grandes desafíos. La motivación al igual que el pensamiento crítico es un tema de creciente utilidad, por ser clave en el aprendizaje para generar interés, deseo y entusiasmo por aprender en conjunto el pensamiento crítico y creativo como la motivación son indispensables para lograr educandos independientes reflexivos y autónomos, siendo motivo para realizar la presente investigación.

Kardoyo et al. (2020) señalan que en Indonesia es fundamental fomentar habilidades de pensamiento crítico y creativo (PCC) en los discentes para enfrentar los desafíos de la revolución industrial 4.0, considerando digitalizar los procesos industriales a través de la interacción de la inteligencia artificial y máquinas.

En Minnesota, Estados Unidos Bowman (2019) manifiesta que se hace necesario la presencia del docente para producir la pasión y el entusiasmo por aprender y el desarrollo del pensamiento crítico y creativo aún con la tecnología avanzando a pasos agigantados, no puede solucionar situaciones cotidianas relacionadas con la calidez humana, las emociones.

En Costa Rica, Mena (2020) refiere que la educación en pensamiento crítico implica el progreso de prácticas especiales de entendimiento y el impulso de condiciones que deben ser aplicadas a tiempo en contextos de la vida diaria, por lo que una formación del pensamiento crítico conduce al mejor desenvolvimiento en la vida de cada estudiante. En Colombia Dorado et al. (2020) realizaron un repertorio de destrezas con las cuales se logró potenciar el proceso educativo en estudiantes ayudándoles a elegir, considerar y ordenar información que ofrecen los profesores, así como solucionar dificultades; evidenciándose que el pensamiento crítico favorece la solución de cualquier conflicto a los que se enfrenten los niños. 
Valencia (2020) en Lima, Perú, refiere que con el uso de la tabla Blackboard los escolares logran incrementar significativamente el pensamiento crítico y que los recursos virtuales deben estar orientados para el bien de la persona y la sociedad. Salazar \& Cabrera (2019) manifiestan que el analizar, procesar información, proponer alternativas de solución, o argumentar, pensar por propia iniciativa, en escolares depende mucho del manejo de las destrezas que utiliza el docente.

El problema de bajo nivel de desarrollo del pensamiento crítico y creativo se evidenció en educación primaria en la Institución Educativa №11015 Comandante Elías Aguirre de Chiclayo, en las escolares del sexto grado con una encuesta, manifestado en limitación en opiniones sin fundamento, sin enjuiciamientos críticos, en lectura sus actitudes son mecánicas con limitadas reflexiones, no demuestran autonomía ni creatividad en sus planteamientos, apreciaciones, así como en los procesos de producción de cuentos, historietas, y todo tipo de textos escritos, en tareas que exigen interpretación y representaciones, de igual modo limitación e inadecuado uso de las TIC en docentes y estudiantes.

Las causas de la situación problemática señalada son: reducida concentración, limitada iniciativa, desmotivación, práctica docente con restringidas técnicas, estrategias en su mayoría obsoletas, reducido uso de la tecnología, escaso reconocimiento de los pequeños logros o esfuerzos, carentes de empatía en la relación entre niñas y son ridiculizadas.

Las consecuencias en las niñas son baja autoestima y calificaciones, opiniones e ideas reducidas, escasa participación, pobreza de vocabulario y razonamiento, falta de reflexión en la toma de decisiones, inseguridad, frustración, indecisión en sus participaciones, limitados logros de progreso. Cabe señalar en circunstancias actuales es difícil contribuir a lo que señala la OCDE (2019) promover una mejor e igual educación para todos, y lo que establece ODS-2030 (CEPAL, 2018), con la intención de asegurar una educación inclusiva, eficaz y de calidad con oportunidades e igualdad de aprendizajes para la vida. Frente a esta problemática surge la pregunta: ¿Cómo un modelo de estrategias motivacionales virtuales contribuye al desarrollo del pensamiento crítico y creativo de las niñas del sexto grado en educación primaria?

El objetivo general es crear un modelo de estrategias motivacionales virtuales para el desarrollo del pensamiento crítico y creativo de las niñas del sexto grado, educación primaria de la institución educativa $\mathrm{N}^{\circ} 11015$ de Chiclayo-2021; los objetivos específicos 
son diagnosticar el nivel de logro de pensamiento crítico y creativo en las estudiantes a partir de un cuestionario; elaborar el diseño de estrategias motivacionales para el desarrollo del pensamiento crítico y creativo; evaluar el modelo de estrategias motivacionales para el desarrollo del pensamiento crítico y creativo.

Se justifica en el aspecto teórico ofreciendo un respaldo en el constructivismo, el cognitivismo, y la teoría de Maslow, que dan un sustento del aprendizaje activo, enfrentando al estudiante a descubrir y buscar soluciones a situaciones de su contexto, apoyados en herramientas tecnológicas y ser reestructurado por procesos mentales al recordar, comprender, aplicar, analizar, evaluar y crear, llevado a cabo en la gestión del aprendizaje con preguntas y repreguntas, para transformar la información, y fortalecer el desarrollo del pensamiento crítico y creativo en las estudiantes.

Surgida la virtualidad rompe por completo el plan de estudios pasando al uso de dispositivos y herramientas, plataformas virtuales utilizar Zoom, Google Meet, Google Classroom entre otras (Molinero \& Chávez, 2019), (MINEDU, 2021) y cada una de ellas con infinidad de herramientas las cuales requiere de estrategias que maneje el docente y las utilice de forma adecuada en el proceso de enseñanza aprendizaje tanto gratuitas como pagadas, de igual forma con los discentes moviéndose en entornos con mucha información.

En este mundo de la virtualidad los estudiantes deben ser orientados para navegar en un ilimitado mundo de información, cual navegante sin rumbo ni destino como lo es el internet, y moverse dentro de un infinito entorno con abundante información, los estudiantes deben ser capaces de analizar y tomar decisiones, para obtener conocimientos (Díaz-Durán \& Svetlichich, 2016) se hace necesario contar con estrategias de análisis y toma de decisiones, para conquistar nuevos ámbitos. Los docentes, en la atmósfera de la formación remota, le urge tener saberes pedagógicos, digitales y tecnológicos además del contenido temático de las áreas curriculares que ellos ya manejan.

\section{ESTRATEGIAS METODOLÓGÍCAS}

\subsection{Tipo y diseño de investigación}

La investigación que se realizó fue de tipo básica, con enfoque cuantitativo, de carácter descriptiva propositiva, porque después del análisis y juicio de la problemática se diseñó un modelo de estrategias motivacionales como propuesta que se puede optar para solucionar o reducir los efectos del problema y lograr satisfacer o superar las necesidades 
o requerimientos en la institución educativa $\mathrm{N}^{\circ} 11015$ “Comandante Elías Aguirre” en Chiclayo (Ñaupas et al., 2018).

El diseño de investigación es no experimental descriptivo propositivo, descriptivo porque partió de un diagnóstico y análisis de la realidad, donde se estableció metas que sirvió de guía para establecer e identificar la problemática de la población en estudio sobre el nivel de desarrollo del pensamiento crítico y creativo. Propositiva porque después de haber realizado el análisis y juicio crítico de la problemática se diseñó un modelo de estrategias motivacionales como propuesta que se puede optar para solucionar o reducir los efectos del problema y lograr satisfacer o superar las necesidades o requerimientos en la institución educativa N¹1015 “Comandante Elías Aguirre” en Chiclayo.

El diseño es de autoría propia, se partió de la realidad y necesidad real donde se localizó la problemática la cual se quiere dar solución acudiendo a teorías que ayudaron a esclarecer. El diseño es abierto, flexible, construido durante el proceso de la investigación, es adaptable al contexto, las situaciones o condiciones que se requiera (Hernández-Sampieri \& Mendoza, 2018).

La siguiente figura muestra el diseño que engloba la problemática y la propuesta de solución.

\section{Figura 1}

\section{Diseño de investigación propositiva}

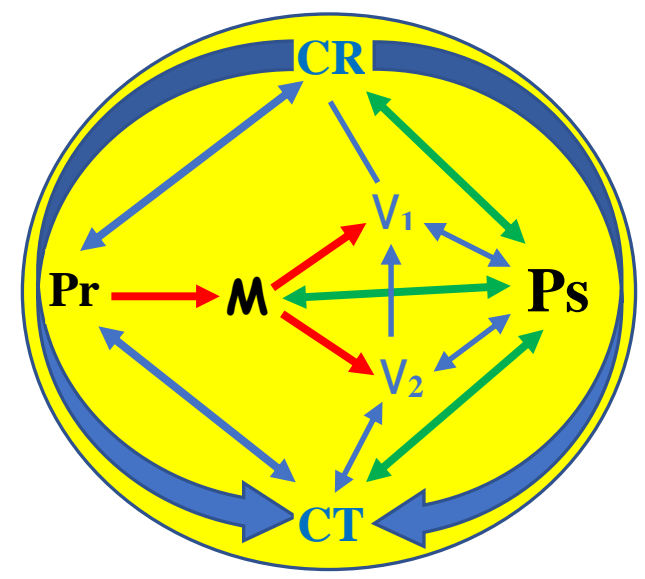

Donde:

CR: Contexto real

Pr: Problema

CT: Ciencia y tecnología

M: Muestra

$\mathrm{V}_{1}$ : Variable1: Pensamiento Crítico Creativo

$\mathrm{V}_{2}$ : Variable 2: Estrategia virtual motivacional

Ps: Propuesta 


\subsection{Variables y Operacionalización}

\subsubsection{Variable dependiente: Pensamiento crítico y creativo}

El pensamiento crítico es un tipo de lógica que tiene que ver con el hecho de cuestionarse (Gunawan \& Nurosyid, 2022) o dar valor evitando impresionarse, emitiendo una postura o determinación con respecto a algo visible, a una idea, actitud o acontecimiento, y para desarrollarlo "exige claridad, precisión, equidad y evidencias... como proceso permite conceptualizar, aplicar, analizar, sintetizar y/o evaluar información recopilada o generada por observación, experiencia, reflexión, razonamiento o comunicación" (Palacios et al., 2017) y (Gao, 2019).

\subsubsection{Variable independiente: estrategias motivacionales}

Son aquellas acciones que están dirigidas para aprender de manera profunda, desplegando sus habilidades y engrandeciendo sus conocimientos mediante el vínculo efectivo de las distintas disciplinas y sus contextos inmediatos (Valenzuela et al., 2018). La utilización de estrategias de aprendizaje se convierten en rutas para lograr un pensamiento que suscite la realización independiente y creadora del estudiante (Betancourth et al., 2019).

\subsection{Población y muestra}

La población estuvo conformada por 134 estudiantes de sexo femenino, de educación primaria de la institución educativa N¹1015 "Comandante Elías Aguirre” de la ciudad de Chiclayo, en el departamento de Lambayeque, distribuidas en 4 aulas, 2 por turno diurno. La población según Cabezas et al. (2018) es considerada un todo y un universo, se puede referir a cualquier conjunto de elementos de los cuales es necesario extraer sus características generales y específicas.

\section{Tabla 1}

Distribución del número de integrantes de la población de estudio del sexto grado de la institución educativa $N^{\circ} 11015$ "Comandante Elias Aguirre"

\begin{tabular}{|c|c|c|}
\hline V Ciclo & Mujeres & total \\
\hline Sección & 35 & 35 \\
\hline B & 36 & 34 \\
\hline C & 33 & 33 \\
\hline D & 32 & 32 \\
\hline Total & & 134 \\
\hline
\end{tabular}

Nota: Nómina de matrícula 2021, directorio de docentes 
La muestra de estudio fue establecida a juicio de la investigadora, conformada por dos aulas del sexto grado que hace un total de 65 niñas con edad entre los 11 y 12 años. Se ha considerado la muestra como un subgrupo de la población determinado de acuerdo con el contexto, necesidades y características propias. (Hernández-Sampieri \& Mendoza, 2018)

\subsection{Técnicas e instrumentos de recolección de datos}

Se aplicó una encuesta que fue concretizada a través de un cuestionario aplicado a las estudiantes de la muestra por medios electrónicos que permitió recolectar datos, fue estructurada considerando la variable de estudio con una escala Likert, sirvió para medir el desarrollo de las habilidades de su pensamiento crítico y creativo. Consta de 30 ítems se consideró 6 dimensiones y sus indicadores, que respondieron a las expectativas de la investigación, de acuerdo a la realidad de la población, con un registro integral, válido y confiable de los hechos y situaciones observables con un propósito que fue estudiado, como lo señalan (Hernández-Sampieri \& Mendoza, 2018).

La técnica de la entrevista se realizó mediante un cuestionario con 12 preguntas que fue aplicada a los docentes de sexto grado que guían a las estudiantes de la muestra, con el propósito de recolectar información sobre las habilidades desarrolladas sobre pensamiento crítico y creativo, así como de las estrategias motivacionales virtuales adquiridas y el impacto que han causado en las estudiantes de la muestra como lo manifiesta (Hernández-Sampieri \& Mendoza, 2018).

Los instrumentos fueron validados por 5 expertos a quienes se le hizo entrega una solicitud adjuntando los documentos pertinentes con la ficha de evaluación respectiva procesados mediante la $\mathrm{V}$ de Aiken.

La confiabilidad fue determinada a través de un piloto sobre el desarrollo del pensamiento crítico y creativo a 15 estudiantes ajenos a la muestra de estudio, los datos recogidos fueron procesados aplicando el alfa de Cronbach cuyo resultado proyecta una confiabilidad de 0,8 el instrumento es confiable y puede ser aplicado en cualquier momento.

\subsection{Procedimientos}

La presente investigación contó con la autorización de la directora de la institución educativa para ingresar a las aulas virtuales, (anexo 05) así como el consentimiento informado a docentes y padres de familia a quienes se les informó y se dio a conocer la 
importancia de la investigación, luego se aplicó los instrumentos por medios virtuales, destinando en un tiempo prudencial de 60 minutos.

\subsection{Método de análisis de datos}

Los datos obtenidos se analizaron estadísticamente para ello se utilizó el programa SPSS v 26, se elaboraron tablas y figuras estadísticas que junto con el fundamento teórico se hizo el análisis respectivo.

\subsection{Aspectos Éticos}

Se tomó en cuenta el código de ética de la Universidad César Vallejo (2020) siguiendo el principio de integridad, respetando los derechos de autor, la propiedad intelectual al citar las fuentes, la confidencialidad, guardando reserva, garantizar la autonomía y bienestar de los participantes.

\section{RESULTADOS}

En el presente capítulo se exteriorizan en forma ordenada los resultados de la investigación relacionados con los parámetros, métricas, criterios; en tablas específicas considerando los objetivos específicos y el objetivo general seguido del respectivo análisis.

\section{Tabla 2}

Nivel de Desarrollo de Pensamiento Crítico y Creativo en las Estudiantes del Sexto Grado de Educación Primaria

\begin{tabular}{|l|c|c|c|c|c|c|c|c|c|c|c|c|}
\hline \multicolumn{1}{|c|}{ Dimensión } & \multicolumn{1}{|c|}{ Recordar } & \multicolumn{2}{c|}{ Comprender } & \multicolumn{2}{c|}{ Aplicar } & \multicolumn{2}{c|}{ Analizar } & \multicolumn{2}{|c|}{ Evaluar } & \multicolumn{2}{c|}{ Crear } \\
\hline \multicolumn{1}{|c|}{ Nivel } & $\mathbf{F}$ & $\mathbf{\%}$ & $\mathbf{F}$ & $\mathbf{\%}$ & $\mathbf{F}$ & $\mathbf{\%}$ & $\mathbf{F}$ & $\mathbf{\%}$ & $\mathbf{F}$ & $\%$ & $\mathbf{F}$ & $\%$ \\
\hline Inicio & 25 & 38,5 & 19 & 29,2 & 24 & 36,9 & 28 & 43,1 & 33 & 50,8 & 36 & 55.4 \\
\hline Proceso & 27 & 41,5 & 30 & 46,2 & 27 & 41,5 & 26 & 40,0 & 24 & 36,9 & 23 & 35.4 \\
\hline $\begin{array}{l}\text { Logro } \\
\text { esperado }\end{array}$ & 11 & 16,9 & 14 & 21,5 & 12 & 18,5 & 9 & 13,8 & 8 & 12,3 & 6 & 9.2 \\
\hline $\begin{array}{l}\text { Logro } \\
\text { destacado }\end{array}$ & 2 & 3,1 & 2 & 3,1 & 2 & 3,1 & 2 & 3,1 & 0 & 0,0 & 0 & 0.0 \\
\hline Total & 65 & 100,0 & 65 & 100,0 & 65 & 100,0 & 65 & 100,0 & 65 & 100,0 & 65 & 100.0 \\
\hline
\end{tabular}

Nota: Aplicación de cuestionario a las estudiantes de la muestra.

En tabla 2; se estimó que, las escolares del 6to grado de primaria 27 niñas de ellas se hallan en proceso sobre la dimensión recordar con el 41,5\%; 25 de ellas que representan el 38,5\% están en inicio, 11 de ellas que hacen el 16,9\% en logro esperado y solo 2 
estudiantes que representan el 3,1\% en logro destacado. Sobre la dimensión comprender 30 alumnas se encuentran en proceso con el 46,2\%; 19 estudiantes que representa el $29,2 \%$ está en inicio, 14 estudiantes con el $21,5 \%$ en logro esperado y solo 2 estudiantes que representan el 3,1\% en logro destacado; así mismo sobre la dimensión aplicar se estimó que las niñas se hallan en proceso con el 41,5\%; el 36,9\% está en inicio, el 18,5\% en logro esperado y solo el 3,1\% en logro destacado. Por consiguiente, sobre la dimensión analizar el $43,1 \%$ se hallan en inicio, el $40 \%$ está en proceso, el 13,8\% en logro esperado y solo el 3,1\% en logro destacado; sobre la dimensión evaluar el 50,8\% se encuentran en inicio; el $36.9 \%$ está en proceso, el 12,3\% en logro esperado y ninguna en logro destacado; además, sobre la dimensión crear el 55,4\% están en inicio, además el 35,4\% se encuentra en proceso, el 9,2\% en logro esperado y ninguna en logro destacado, como se aprecia que, del total de estudiantes del sexto grado de educación primaria que fueron evaluadas, se encontró en su mayoría sobre el desarrollo de pensamiento crítico y creativo se hallan en proceso e inicio, y muy pocas niñas han logrado ubicarse en logro esperado y logro destacado.

\section{DISCUSIÓN}

Después de realizar el proceso estadístico; la interpretación de los datos se realizó la discusión de lo encontrado partiendo como base fundamental de los objetivos de la investigación; además, se tiene en cuenta estudios y teorías que afirman lo hallado.

El objetivo general fue crear un modelo de estrategias motivacionales virtuales para el fortalecimiento del desarrollo del pensamiento crítico y creativo de las niñas del sexto grado, educación primaria de la institución educativa $\mathrm{N}^{\circ} 11015$ de Chiclayo-2021, según los resultados la mayoría de niñas se encuentran en proceso e inicio, de lo expuesto, al respecto el estudio de Demirhan \& Köklükaya, (2014) manifiestan que los docentes realizan el esfuerzo de poder incrementar el pensamiento crítico lo cual se tiene en cuenta en la preparación de los futuros docentes tomando como base una programación para desarrollar el pensamiento crítico, y así formar a estudiantes que represente y transforme su realidad. El autor considera que se debe plantear estrategias o modelos para robustecer el pensamiento crítico de los estudiantes pues la capacidad de todo ser humano se refleja en un contexto donde el individuo tiene que solucionar problemas que se le presenten.

Özelçi \& Çalışkan (2019) en sus estudios representa como base fundamental el desarrollo del pensamiento crítico; el cual es aplicado desde el currículo nacional en Turquía al 
incluir de forma obligatoria en los planes de estudio, obteniendo resultados y avances tanto en el desempeño docente como en los futuros docentes para que salgan a desempeñar su labor con conocimientos y habilidades claras. Se plantea que en toda escuela los docentes deben plantear estrategias o modelos que promuevan dichas habilidades; el pensamiento crítico en toda persona se pondrá en acción cuando este determine un problema; para solucionarlo, partiendo de una realidad concreta, teniendo en cuenta lo llevará a codificar sucesos nuevos y una flexibilidad mental; es decir lo llevará a codificar sucesos nuevos y a obtener las nuevas experiencias que ha adquirido significativamente.

Asimismo, Díaz-Larenas et al. (2019) considera que la crítica constructiva en todo ser humano es un compromiso en la educación; elemento debe ser asumido con responsabilidad por el sistema educativo, para lograr tener una sociedad que se desempeñe reflexionando antes de actuar, respetando la opinión de los demás. Por otro lado, llega a la conclusión que la educación en todos sus niveles, áreas y actores asuma la responsabilidad de incluir en el currículo el desarrollar el pensamiento crítico, con la finalidad de lograr seres humanos sensibilizados que actúan tomando en cuenta a los demás y participen por una sociedad más culta y humana.

El autor considera de suma importancia desarrollar el pensamiento crítico; mediante estrategias o modelos, que le permitan al discente impulsar un profundo análisis de la realidad para tomar decisiones acertadas y crecer en experiencias personales $\mathrm{y}$ profesionales.

Castellaro \& Peralta (2020) en su estudio considera el compromiso que tiene todo docente en asumir una educación crítica donde es facilitador, mediador que genera el andamiaje y permita al estudiante crear sus propias formas de tomar decisiones, de resolver situaciones, que involucren sus juicios críticos para cambiar y seguir aprendiendo, que se desempeñe de forma reflexiva respetando la opinión de los demás y cumpla su responsabilidad en todos los niveles y áreas para transformar la sociedad. El autor concluye que se debe tener en cuenta estrategias que permitan desarrollar la capacidad crítica de los estudiantes, siendo la criticidad un compromiso que debe ser asumido por la educación con un currículo transversal.

Según Palacios et al. (2017) el pensamiento crítico para desarrollarlo "exige claridad, precisión, equidad y evidencias... como proceso permite conceptualizar, aplicar, analizar, 
sintetizar y/o evaluar información recopilada o generada por observación, experiencia, reflexión, razonamiento o comunicación”. Para el autor el pensamiento crítico tiene mucho que ver con el razonamiento, la reflexión, sin tener que conmoverse dejando de lado la pasión, es necesario tomar una actitud firme bien analizada y reflexiva, que sea definitiva y contundente.

Primer objetivo específico diagnosticar el nivel de pensamiento crítico y creativo en las estudiantes del sexto grado de educación primaria. Se toma en cuenta los estudios de Ramírez (2017) en la investigación con la propuesta del uso de las redes sociales para mejorar el pensamiento crítico de los estudiantes, toma como resultados que los estudiantes se encuentra en un nivel bajo y para ello, el autor, propone superar estas deficiencias al hacer una motivación a partir de un video sobre el cual los niños dramatizan responden preguntas de análisis y reflexión para finalmente trabajar material de lectura. En conclusión, el análisis y la reflexión a partir de realizar preguntas son clave para desarrollar el pensamiento crítico de los educandos en todas sus habilidades para tener mejores desempeños.

El autor sustenta que el progreso conveniente del pensamiento crítico en el ser humano; no sólo es necesario en el aspecto académico; sino también en su vida propia y profesional; donde se convierta en visionario con características imaginativas, abiertas, flexibles y con una auto confianza de potencial y acciones colectivas.

Vargas y Acuña (2020) consideran que el pensamiento crítico en toda persona es de suma importancia en cualquier aspecto de su vida, ya que este le permite ser más imaginativo y mirar mejor el panorama de la situación a la que se está enfrentando, esto también al estudiante le permite tomar decisiones correctas en su vida personal y profesional. Se compara este estudio con el presente trabajo de investigación al destacar el alcance, influencia y la importancia del pensamiento crítico en la investigación.

Segundo objetivo específico elaborar el diseño de estrategias motivacionales para el desarrollo del pensamiento crítico y creativo.

La motivación y el pensamiento crítico y creativo son habilidades indispensables en esta época y "en la actualidad existe una gran preocupación por encaminar y potenciar en los estudiantes ciertas habilidades de pensamiento superior que les será útil en su aprendizaje" Tapia \& Castañeda (2021) y en su actuar en el futuro al tomar decisiones, al resolver problemas de su vida cotidiana. 
Los autores hacen referencia a tomar en cuenta tanto el pensamiento crítico como la motivación dentro de la planificación y la gestión del aprendizaje de los educandos, muy a tener en cuenta por los docentes por ser habilidades imprescindibles para el futuro de cada individuo y de la sociedad al lograr resolver situaciones de su contexto.

Belmonte (2018) propone una estrategia de didácticas de las redes sociales virtuales para el desarrollo del pensamiento crítico en estudiantes de educación media, utilizó la técnica de observación directa e indirecta mediante la videograbación para realizar el análisis detallado de episodios realizados en clase, y ver el actuar de los docentes. En conclusión, se determina que este programa llevó muchos efectos positivos en los estudiantes, en la cual se concreta que se desarrolla el pensamiento crítico.

El autor considera que se deben aplicar estrategias emocionales que provoquen una productividad, armonía y solución de problemas rutinarios, esto dignifica que, educar emocionalmente es ser empáticos y comprender a los demás, mostrando conformidad al percibir las emociones que está sintiendo, asimismo es precisar límites, brindar modelos apropiados y de socialización, también permite respetar a los demás. Para despertar las motivaciones de los estudiantes es indispensable tener en cuenta el interés del estudiante, como los temas de se consideren atractivos y los contenidos que le gustaría ver en clase y el cómo los realiza.

Así Fagúndez et al. (2015) al realizar una experiencia con la aplicación de su programa en una institución educativa de Uruguay, donde la motivación y los desafíos a los que se enfrenta a los estudiantes, estos llegan a proponer, concretar ideas en actividades, con ello generaron cambios profundos en el ámbito del pensamiento de los niños desde la razón, para llevarlo hasta el pensamiento crítico. En conclusión, los estudiantes muestran y encuentran oportunidades para solucionar situaciones problemas de su entorno de forma creativa.

El autor considera que las estrategias motivacionales son importantes porque facilitan el logro de los aprendizajes, es decir traspasa en la búsqueda de la información profunda, apropiándose de las necesidades e intereses de los educandos y su contexto real. Por lo tanto, se observa cómo el estudiante aprende y que debe lograr la autonomía y la autorregulación.

Al respecto Villarini (2004) citado por Bezanilla-Albisua et al. (2018) manifiesta que se necesita de un "aprendizaje auténtico", donde es necesario incluir actividades 
reveladoras, estimuladoras, juiciosas, colaborativas y fortalecedoras que englobe el pensamiento crítico. Además se toma en cuenta la investigación de Delgado et al. (2020) quienes manifiesta que la educación en estos momentos pasa por dificultades lo cual se agrava por una etapa digital que ha producido cambios en docentes, estudiantes, y en todos los seres humanos por la forma de comunicarse de participar, de pertenencia de gestionar, de compartir o recrearse por todo ello la educación necesita hacer un esfuerzo para satisfacer esta necesidad.

Se toma en cuenta estos estudios para diseñar las estrategias motivacionales por presentarse las dificultades que se está viviendo con la pandemia Covid-19, donde se cambió lo presencial por lo virtual y se hace aún más complicado mantener la motivación y el entusiasmo de las estudiantes para mantener su atención, emoción y concentración. Tercer objetivo específico evaluar el modelo de estrategias motivacionales para el desarrollo del pensamiento crítico y creativo.

Para poder evaluar, se toma en cuenta los resultados, partiendo de una realidad concreta encontrada que revelan sobre la dimensión aplicar, y la dimensión comprender la mayoría de las niñas se ubica en proceso e inicio.

Estos resultados permiten evaluar la importancia de un modelo de estrategias motivacionales para el desarrollo del pensamiento crítico y creativo. Al compararlos con estudios similares se puede valorar la importancia de un modelo de estrategias motivacionales virtuales para el fortalecimiento del desarrollo del pensamiento crítico y creativo, sobre el cual Priawasana, et al. (2020), consideran que en Indonesia tiene importancia el desarrollo del pensamiento crítico porque este contribuye a la toma de decisiones y la solución de dificultades de su realidad. Se afirma que el pensamiento crítico está presente en la conciencia como una luz para facilitar tomar decisiones y soluciones de situaciones o problemas que se le presente en el diario vivir todo esto a través de programas o estrategias aplicados por los docentes en las sesiones de aprendizajes.

Canese (2020) considera que el pensamiento crítico, debe ser desarrollado; sino que este tiene que ser desarrollado o motivado por algunas estrategias que el docente aplique y que permita formular una reflexión o tomar una iniciativa propia en una crítica meditada en un cambio social. 
El autor considera que los docentes pueden transmitir motivos a los estudiantes para reforzar y reorientar la creación de proyectos personales y profesionales; que les permita despertar el interés y sentimiento de competencias creativas, para solucionar aspectos personales en la toma de decisiones de tal manera que el estudiante se sienta satisfecho de lograr sus objetivos, tanto como esto sea útil y provechoso para el aspecto laboral y educativo.

También Hernández et al. (2015) manifiestan que el pensamiento creativo al ser manipulado por algunas estrategias utilizadas por el docente, esto se ve reflejado al emplear el talento intelectual y al ser juiciosos, y se desarrolla al aprovechar la facultad intelectual, además un ser racional que filosofa de su entorno tiene tendencia a cuestionarse y a buscar respuestas de su realidad, asimismo, tolera un bien que le permite el entendimiento de la naturaleza y lo que se desea conseguir al evaluar y validar su trabajo e inventiva producto de su imaginación y originalidad.

El autor reafirma la postura de que el docente debe gestionar un aprendizaje con productos creativos, y hacer que los estudiantes hagan realidad sus ideas, sus sueños, con miras a solucionar situaciones problemáticas.

Cardero \& Arencibia (2019) y (Aylar-Çankaya, 2020) hacen referencia que el docente debe utilizar estrategias para impulsar el pensamiento creativo, en sus estudiantes, mencionan que un estudiante creativo mejora o produce ideas nuevas obteniendo resultados o productos originales, siendo la creatividad una capacidad realizable que existe en los seres humanos para engendrar soluciones únicas de forma reflexiva e imaginativa expresando emotividad, en función a una dimensión cognitiva, motora y afectiva.

Los autores toman al docente como agente de cambio que propone y utiliza estrategias en las sesiones de aprendizajes para guiar y facilitar la toma de decisiones y soluciones de situaciones o problemas y la que se les presenta en el diario vivir a los estudiantes.

\section{CONCLUSIONES}

Partiendo de la problemática realizada en la investigación se encaminó a diseñar y crear un modelo de estrategias motivacionales virtuales para el fortalecimiento del desarrollo del pensamiento crítico y creativo de las niñas del sexto grado, educación primaria de la institución educativa $\mathrm{N}^{\circ} 11015$ de Chiclayo-2021, tomando en cuenta que el pensamiento crítico y creativo como la habilidad del ser humano de formar nuevas ideas para satisfacer 
necesidades o resolver problemas contextuales a partir de un cuestionamiento, reflexión, formulación de juicios y planteamientos que se realice de forma interna. Mediante este resultado se toma en cuenta el objetivo general, de acuerdo con los resultados, donde se puede entender la importancia del modelo de estrategias motivacionales virtuales para el fortalecimiento del desarrollo del pensamiento crítico y creativo.

El diagnóstico del nivel de desarrollo pensamiento crítico y creativo de las estudiantes que en su mayoría se encontró en inicio y proceso, refleja una realidad concreta de las estudiantes del sexto grado de educación primaria.

A partir del diagnóstico se elaboró el diseño de estrategias para el desarrollo del pensamiento crítico y creativo, por lo tanto, cobra importancia diseñar las estrategias motivacionales virtuales que fortalezcan el pensamiento crítico y creativo de las estudiantes.

Se estableció evaluar el modelo de estrategias motivacionales virtuales para el desarrollo del pensamiento crítico y creativo, por lo tanto, se puede dar fe de la importancia del modelo de estrategias que permite que las estudiantes se caractericen por ser pensadoras hábiles y sofisticadas.

\section{PROPUESTA}

La propuesta titulada Estrategias motivacionales virtuales para desarrollar el pensamiento crítico y creativo, tiene como respaldo las teorías constructivistas, socio cultural basada en la teoría del conocimiento constructivista sustentado por Vygotsky, Ausubel, Bruner, la teoría de las necesidades de Maslow y la taxonomía de Bloom.

El constructivismo en educación hace sus contribuciones al afirmar que el aprendizaje es un proceso de desarrollo de destrezas cognitivas, afectuosas y sociales aprehendida según los niveles de madurez del estudiante, lo cual implica que la información que se le brinde sea significativa, novedosa para causar desequilibrio, ser asimilada, acomodada y aprendida (Ortiz 2015) produciendo un cambio y adaptación al entorno en un proceso de interacción social.

Se ha considerado también las características y necesidades de las estudiantes participantes en la muestra, el contexto donde se ubica la institución educativa, el logro en las experiencias de aprendizaje realizadas como docente del grado, los procesos con las que se puede desarrollar las competencias, capacidades y habilidades de las estudiantes además de los resultados obtenidos en base a los datos recogidos a través de 
la administración de instrumentos de investigación específicos, debidamente validados y confiables con el cual se diagnosticó la realidad problemática en el nivel de logro del desarrollo del pensamiento crítico y creativo de las estudiantes

Esta propuesta consiste en realizar una lectura con actitud analítica y crítica, para lo cual es necesario formular preguntas y repreguntas adecuadas, desde las más simples y sencillas hasta lograr hacer reflexionar y argumentar respuestas mediante un constante análisis y evaluación para llegar a la toma de decisiones, donde interviene la motivación con el uso de las herramientas tecnológicas como Google drive, Google Meet, YouTube, Lucichard, Educaplay, Canva, Xmind, videos, Patlet, pizarra Jamboard, blog, que le permiten compartir de manera sincrónica y asíncrona, mostrando la importancia y desarrollo del pensamiento crítico, así como el mejoramiento del conocimiento y las actuaciones de las estudiantes aportando en un trabajo en equipo o colaborativo con opiniones, reflexiones, creaciones, ideas a mejorar o evidenciar sus actividades virtuales que se les proponga. En el siguiente esquema se muestra la propuesta. 


\section{Modelo de estrategias motivacionales virtuales para el pensamiento crítico y creativo}

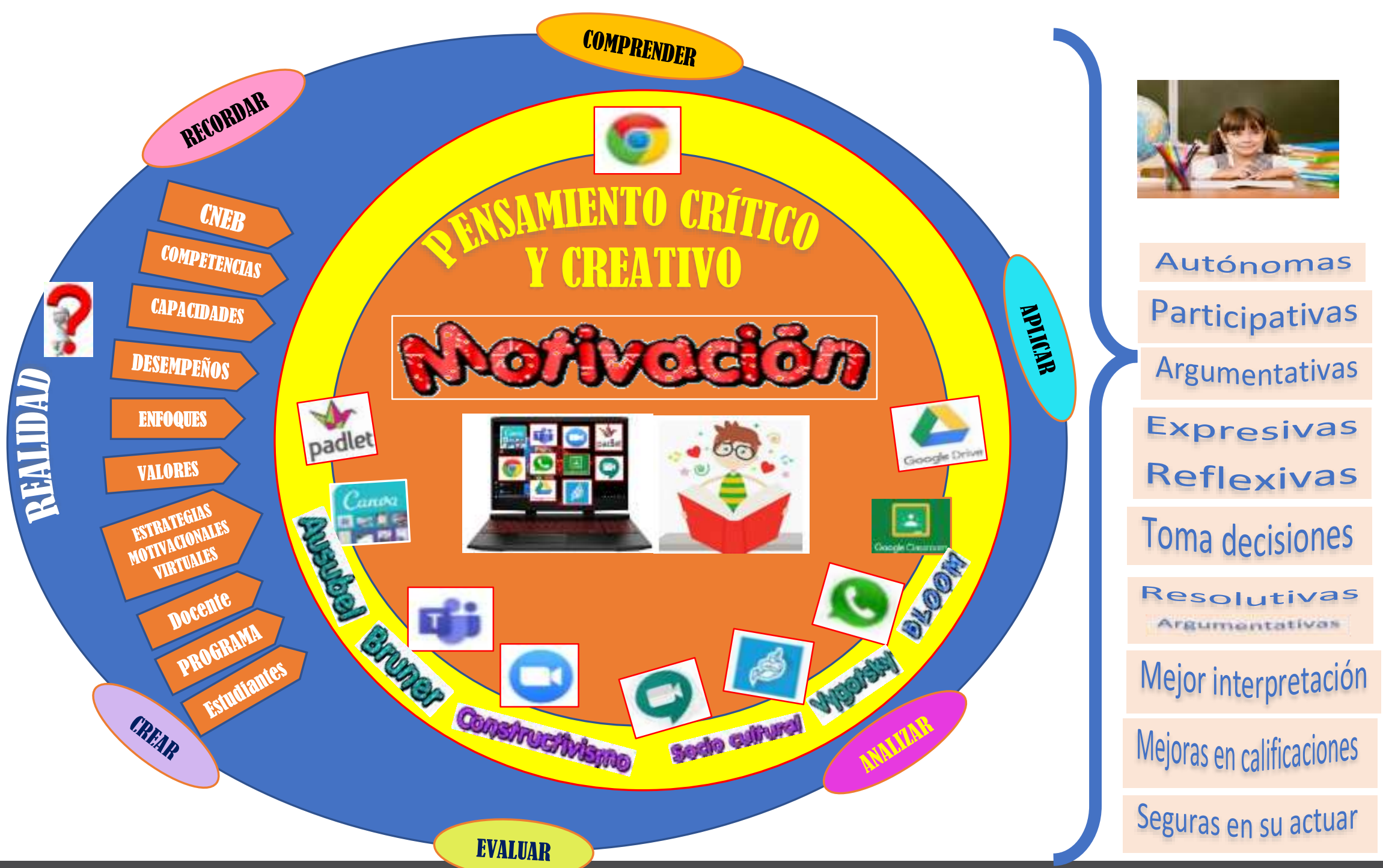

Ciencia Latina Revista Científica Multidisciplinar, Ciudad de México, México.

ISN 2707-2207 / ISSN 2707-2215 (en línea), enero- febrero, 2022, Volumen 6, Número 1. https://doi.org/10.37811/cl_rcm.v6i1.1769 p 3806 


\section{REFERENCIAS BIBLIOGRAFICAS}

Aylar-Çankaya, E. (2020). Investigating Flexibilities of the Classroom Teachers for Four Operations in the Basis of Different Strategies. Kuramsal Eğitimbilim, 13(4), 646662. https://doi.org/10.30831/akukeg.646023

Betancourth, S., Tabares, Y., \& Martínez, V. (2019). Programa de intervención en debate crítico sobre el pensamiento crítico en universitarios. Educación y Humanismo, 22(38), 1-17. https://doi.org/10.17081/eduhum.22.38.3577

Bezanilla-Albisua, M., Poblete-Ruiz, M., Fernández-Nogueira, D., Arranz-Turnes, S., \& Campo-Carrasco, L. (2018). El Pensamiento Crítico desde la Perspectiva de los Docentes Universitarios. Estudios Pedagógicos (Valdivia), 44(1), 89-113. https://doi.org/10.4067/S0718-07052018000100089

Bowman, R. (2019). A New Story About Teaching and Learning. The Clearing House: A Journal of Educational Strategies, Issues and Ideas, 92(3), 112-117. https://doi.org/10.1080/00098655.2019.1613339

Cardero, D., \& Arencibia, M. (2019). Habilidades creativas en equipos de desarrollo de software. Cuadernos de Educación y Desarrollo, 111.

Castellaro, M., \& Peralta, N. (2020). Pensar el conocimiento escolar desde el socioconstructivismo: Interaccion, construccion y contexto. Perfiles Educativos, 42(168), 140-156. https://doi.org/10.22201/IISUE.24486167E.2020.168.59439

CEPAL. (2018). La Agenda 2030 y sus Objetivos de Desarrollo Sostenible. In Revista de Derecho Ambiental (Issue 10). https://doi.org/10.5354/0719-4633.2018.52077

Delgado, J., Garcia, A., \& Martinez, O. (2020). La influencia del conectivismo para el uso de las TIC en el proceso enseñanza-aprendizaje. Akrab Juara, 5(1), 43-54. http://www.akrabjuara.com/index.php/akrabjuara/article/view/919

Demirhan, E., \& Köklükaya, N. (2014). The Critical Thinking Dispositions of Prospective Science Teachers. Procedia - Social and Behavioral Sciences, 116(2006), 1551-1555. https://doi.org/10.1016/j.sbspro.2014.01.433

Díaz-Durán, M., \& Svetlichich, M. (2016). Nuevas herramientas tecnológicas en la educación superior. Proyecciones: (La Plata), 11(11), 93-149. https://dialnet.unirioja.es/servlet/articulo?codigo=7111293\&info=resumen\&idio ma=ENG\%0Ahttps://dialnet.unirioja.es/servlet/articulo?codigo $=7111293 \&$ info $=$ resumen\&idioma $=$ SPA\%0Ahttps://dialnet.unirioja.es/servlet/articulo?codigo $=71$ 
11293

Díaz-Larenas, C., Ossa-Cornejo, C., Palma-Luengo, M., Lagos-San Martín, N., \& Boudon, J. (2019). El concepto de pensamiento crítico según estudiantes chilenos de pedagogía. $\quad$ Sophía, 268-277. https://doi.org/10.17163/soph.n27.2019.09

Dorado, A., Ascuntar, J., Garces, Y., \& Obando, L. (2020). Programa de estrategias de aprendizaje para estudiantes de una institución educativa. Praxis \& Saber, 11(25), 75-95. https://doi.org/10.19053/22160159.v11.n25.2020.9272

Gao, Y. (2019). Analytical Reading as an Effective Model for Enhancing Critical Thinking. 329(Iccessh), 318-322. https://doi.org/10.2991/iccessh-19.2019.72

Gunawan, Y., \& Nurosyid, F. (2022). Analysis of High School Students ' Critical Thinking Level Based on Logical Arguments. 12(1), 137-145. https://doi.org/10.1115/italienisch.v12i1.181

Hernández-Sampieri, R., \& Mendoza, C. (2018). Metodología de la investigación: las tres rutas cuantitativa, cualitativa y mixta. In Mc Graw Hill (Vol. 1, Issue Mexico).

Hernández, I., Alvarado, J., \& Luna, S. (2015). Creatividad e innovación : competencias genéricas o transversales en la formación profesional. Revista Virtual Universidad Catolica Del Norte, 135-151. https://revistavirtual.ucn.edu.co/index.php/RevistaUCN/article/view/620/1155

Kardoyo, Nurkhin, A., Muhsin, \& Pramusinto, H. (2020). Problem-Based Learning Strategy: Its Impact on Students' Critical and Creative Thinking Skills. European Journal of Educational Research, 9(3), 1141-1150. https://doi.org/10.12973/eujer.9.3.1141

Mena, A. (2020). Critical Thinking for Cvic Life in Elementary Education: Combining Storytelling and Thinking Tools. Revista Educación, 44, 23-43. https://doi.org/10.15517/revedu.v44i2.39699

MINEDU. (2021). Rvm $N^{\circ}$ 234-2021-Minedu. 1-29.

Molinero, M., \& Chávez, U. (2019). Herramientas tecnológicas en el proceso de enseñanza-aprendizaje en estudiantes de educación superior. RIDE Revista Iberoamericana Para La Investigación y El Desarrollo Educativo, 10(19). https://doi.org/10.23913/ride.v10i19.494

Naupas, H., Valdivia, M., Palacios, J., \& Romero, H. (2018). Metodología de la 
investigación cuantitativa-cualitativa y redacción de la tesis. In Journal of Chemical Information and Modeling (Vol. 53, Issue 9). https://doi.org/10.1017/CBO9781107415324.004

OCDE. (2019). El trabajo de la OCDE sobre Educación y competencias. Oecd, 1, 1-28.

Özelçi, S., \& Çalışkan, G. (2019). What is critical thinking? A longitudinal study with teacher candidates. International Journal of Evaluation and Research in Education, 8(3), 495-509. https://doi.org/10.11591/ijere.v8i3.20254

Palacios, W., Álvarez, M., Moreira, J., \& Morán, C. (2017). Una mirada al pensamiento crítico en el proceso docente educativo de la educación superior. Edumecentro, 9(4), 194-206.

Salazar, D., \& Cabrera, X. (2019). Estrategias Didécticas Para Fortalecer El Pensamiento Crítico En Estudiantes De Tercer Grado De Primaria En Institucion De Chiclayo. 12, 1-9.

Tapia, M., \& Castañeda, E. (2021). Percepción futurista sobre pensamiento crítico en la nueva era. Revista Innova Educación, 4(2), 45-61. https://doi.org/10.35622/j.rie.2022.02.003

Universidad César Vallejo. (2020). Código de etica en investigacion. Vicerrectorado de Investigación, 1-16. https://es.scribd.com/document/509036811/Codigo-deetica-en-Investigacion-UCV

Valencia, C. (2020). Educación virtual en el pensamiento crítico en los estudiantes de una universidad privada de Lima, 2020. 0-125.

Valenzuela, J., Muñoz, C., \& Montoya, A. (2018). Estrategias motivacionales efectivas en profesores en formación. Educação e Pesquisa, 44(0), 1-20. https://doi.org/10.1590/s1678-4634201844179652

Villarini, Á. (2004). Teoría y pedagogía del pensamiento crítico. Perspectivas Psicológicas, 3-4, 35-42. http://pepsic.bvsalud.org/pdf/pp/v3-4/v3-4a04.pdf 